\title{
Maderas de zonas áridas de México, poder calorífico por ATG-ATD y mediante bomba calorimétrica
}

\section{Woods from arid zones of Mexico, calorific power from TCA-DTA and calorimetric pump}

\author{
Enrique Barrera-Calval* Federico González.', Carlos David Hernández-Pérez.' ', Gloria Martínez², \\ Lázaro Huerta-Arcos. ${ }^{3}$, Ricardo Rosas-Cedillo' y Víctor Rentería-Tapia ${ }^{4}$
}

1 Universidad Autónoma Metropolitana, Iztapalapa, Área de Ingeniería en Energía, IPH, CBI. Ciudad de México, México.

2 Instituto Tecnológico de Celaya, Departamento de Ingeniería Química. Celaya, Guanajuato, México

\author{
3 Universidad Nacional Autónoma de México. \\ Instituto de Investigaciones en Materiales. Ciudad \\ de México, México. \\ 4 Universidad de Guadalajara. Departamento de \\ Ciencias Naturales y Exactas. Centro \\ Universitario de los Valles. Jalisco, México.
}

\section{RESUMEN}

En este trabajo se determina el poder calorífico de maderas de huizache (Acacia farnesiana), maguey (Agave salmiana), nopal (Opuntia streptacantha), garambullo (Myrtillocactus geometrizans) y cardón (Pachycereus sp) que provienen de zonas áridas de México y que suelen utilizarse para el calentamiento de agua y cocción de alimentos en el medio rural. La determinación de los poderes caloríficos se realizó por medio de las técnicas de análisis térmico gravimétrico y la técnica de análisis térmico diferencial, ATG-ATD, que es similar a la calorimetría diferencial de barrido (DSC), y mediante una bomba calorimétrica de combustión. En general, los resultados mostraron que el poder calorífico estimado por análisis (ATG-ATD) resulta en todos los casos ligeramente menor que lo estimado con bomba calorimétrica, pero en general son muy próximos entre sí. La máxima variación entre dichos valores en $\pm 5 \%$ se asocia en un intervalo dentro de la incertidumbre de las mediciones realizadas. Para la madera de nopal se encontró que el poder calorífico estimado por ATG-ATD alcanza 11 $275 \mathrm{~kJ} / \mathrm{kg}$, resultando muy parecido al valor determinado con bomba calorimétrica, $11605 \mathrm{~kJ} / \mathrm{kg}$. En los otros casos estudiados se encuentra una mayor discrepancia entre los valores medidos. El análisis elemental de las maderas y de sus cenizas permite la determinación del parámetro energético ambiental denominado intensidad de carbono de las maderas. Este se estima por medio de espectroscopía de fotoelectrones emitidos por rayos X (XPS), lo que indica que no hay evidencia de metales tóxicos como los provenientes del carbón; esto demuestra que estas maderas son un producto sutentable y renovable cuando están disponibles para la combustión y obtención de energía calorífica.

PALABRAS CLAVE: análisis térmico diferencial, bomba calorimétrica, intensidad de carbono, poder calorífico.

\section{ABSTRACT}

The calorific power of wood of huizache (Acacia farnesiana), maguey (Agave salmiana), nopal (Opuntia streptacantha), garambullo (Myrtillocactus geometrizans) and cardon (Pachycereus sp) from arid areas of Mexico, which are often used for heating water and cooking food in rural areas, in this work are presented. The calorific power determination was carried out by means a calorimetric combustion pump and of a gravimetric thermal analysis, GTA and differential thermal analysis technique, DTA, which is quite similar to the differential scanning calorimetry (DSC). In general, the results showed that the calorific value estimated by analysis (GTA-DTA) is in all cases slightly lower than those estimated with a calorimetric pump, but in general, both results are very close between each other. Maximum variation between these values is within $\pm 5 \%$, which is associated in a range within the uncertainty of the measurements made. For the nopal wood, it was found that the calorific value, estimated by GTA-DTA, reaches $(11275 \mathrm{~kJ} / \mathrm{kg})$, resulting very similar to the value determined with a calorimetric pump, (11 $605 \mathrm{~kJ} / \mathrm{kg}$ ). In the other cases studied a greater discrepancy between the measured values was found. The elementary analysis of the woods and their ashes estimated by means of X-ray photoelectron spectroscopy (XPS), allows the determination of the carbon intensity of the woods, and indicates that there is no evidence of toxic metals such as those from coal, which shows that these woods are a sustainable and renewable product, when they are available for combustion and heat energy obtainment.

KEYWORDS: differential thermal analysis, calorimetric pump, carbon intensity, calorific power. 


\section{INTRODUCCIÓN}

En las áreas urbanas de México se utiliza gas natural o propano líquido, LP, para cocinar o calentar alimentos y agua. Sin embargo, en las áreas rurales la escena es completamente diferente, pues un alto porcentaje de su población utiliza madera, residuos agrícolas o desechos orgánicos para proporcionar la energía de esas aplicaciones. Debido a su ubicación y a sus características de clima y orografía, México tiene diferentes tipos de recursos forestales (Instituto Nacional de Estadística y Geografía [Inegi] 2018). Las siguientes cifras son suficientes para explicarlo: del área total de tierra (ATT) de México, casi $2 \times 10^{6} \mathrm{~km}^{2}, 72 \%\left(1.42 \times 10^{6} \mathrm{~km}^{2}\right)$ está dedicado a diferentes usos forestales. El país tiene varios ecosistemas de bosques naturales, los principales tipos son: los bosques templados fríos (bosques de coníferas y de hoja ancha) que representan $15.6 \%$ de ATT $\left(3.04 \times 10^{5} \mathrm{~km}^{2}\right)$; Las selvas tropicales representan $13.5 \%$ de ATT $\left(2.64 \times 10^{5} \mathrm{~km}^{2}\right)$, y la vegetación en las zonas áridas representan $29.9 \%$ de AT'T $\left(5.85 \times 10^{5} \mathrm{~km}^{2}\right)$. Como se señaló, la mayor ATT corresponde a zonas áridas, ya que México es un país de enorme extensión territorial, pero con una grave escasez de agua. Una pequeña área en el sureste de México representa 66\% del agua doméstica; mientras que el gran total del país solo tiene un promedio anual de precipitaciones de $758 \mathrm{~mm}$ (Banco Mundial [BM] 2018).

Las zonas áridas con vegetación donde se asientan miles de comunidades rurales del país representan las áreas de interés que le da aprovechamiento, poco evaluado, a la combustión de la leña en estas regiones (58.5 millones de hectáreas). Es evidente que existe una gran superficie de terreno no apto para la agricultura, pero sí con una importante población de plantas de zonas áridas donde destacan poblaciones de nopal, maguey, garambullo, mezquite, huizache, cardón y otras diferentes cactáceas que suelen utilizarse para la combustión, para el calentamiento de agua y para la cocción de alimentos (Secretaría de Agricultura, Ganadería, Desarrollo Rural, Pesca y Alimentación [Sagarpa] 2018). Además, estas plantas tienen también un amplio potencial en diferentes aplicaciones médicas y alimenticias. Por ejemplo, la hoja del nopal es un producto usual en la dieta del mexicano que en los últimos tiempos ha tomado relevancia por sus propiedades alimenticias y propiedades dietéticas que ayudan a mantener una buena nutrición y salud estomacal al ser humano. Al contener altas proporciones de fibra ayuda a la digestión, evita el estreñimiento y previene cáncer del área estomacal, según los consumidores de este cactus y el estudio de Kamble, Prashant, Ranveer y Sahoo (2017).

La combustión de los productos o residuos agrícolas para la población rural que usualmente vive en zonas áridas es una actividad común y de subsistencia popular, en virtud de que la compra de gas propano, cuyo costo es aproximadamente USD $1 / \mathrm{kg}$, es muy difícil de conseguir dado el bajo poder adquisitivo de dicha población asentada en esas regiones del país. En estos lugares las personas aprovechan de la combustión de la madera, aun de zonas áridas, para la cocción de sus alimentos y para el calentamiento de su agua de baño, consumiendo típicamente hasta $5 \mathrm{~kg}$ por persona por día.

Los autores del presente estudio consideran que los recursos maderables provenientes de zonas áridas no han sido evaluados totalmente tanto en cantidad como en sus valores de poder calorífico en detalle. La tierra árida no es adecuada para la agricultura, pero hay una variedad de plantas xerofíticas, destacando las poblaciones de nopal (Opuntia streptacantha), maguey (Agave salmiana), garambullo (Myrtillocactus geometrizans), mezquite (Prosopis laevigata), huizache (Acacia farnesiana) y otros cactus, con potencial calorífico desconocido o no estimado en diferentes condiciones, pero ampliamente utilizados para combustión, calentamiento de agua y cocción.

Con respecto a los materiales de madera en zonas áridas, aún en la actualidad están recibiendo poca importancia, a pesar de que se obtiene una gran cantidad de productos de la flora de las zonas secas como el xito, el caucho, las fibras textiles, los extractos como la jojoba (nativa del desierto de Sonora en el noroeste de México) y el nopal, que es un vegetal típico y extendido de los sitios áridos que durante siglos ha dado una variedad de productos valiosos al país. 
Para garantizar la protección ecológica de las regiones más representativas, el gobierno mexicano creó una red de áreas naturales protegidas, que son partes terrestres o acuáticas de los diferentes ecosistemas y su biodiversidad. A pesar de tales programas, las regiones áridas se olvidan en gran medida y se utilizan poco, a pesar de que en ellas la combustión de la madera se utiliza en exceso. Es por ello que este estudio podría proporcionar claridad sobre el potencial energético de las maderas provenientes de estas regiones, tomando en cuenta la regeneración y la protección de la especie. La utilización de leña de diferentes plantas que se obtienen de las zonas áridas es muy común y este hecho debe ser ampliamente conocido. Lejos de preocuparnos, y como clama la organización ecológica internacional Greenpeace (2020), esto debe alentar programas gubernamentales y privados para reforestar dichas regiones y así aprovechar la facultad de reproducción de estas plantas de zonas áridas que, aunque crecen a baja tasa por la limitación de agua en tales regiones, son potencialmente importantes productores de volúmenes apreciables de leña para la población asentada en esas comunidades.

Para conocer la eficiencia de la biomasa de madera como combustible, se requiere conocer sus propiedades termoenergéticas, tal como el poder calorífico, el cual está relacionado con la energía liberada en el proceso de combustión (Musule et al., 2018). Similarmente, la estimación de la composición química de las cenizas derivadas de la combustión de la biomasa de madera es de gran importancia para verificar su sustentabilidad.

El presente trabajo pretende dar a conocer los poderes caloríficos de tales plantas típicas que son naturales de estas regiones, dado que poco más de $10 \times 10^{6}$ de pobladores de zonas áridas de México queman madera de plantas. Adicionalmente, se compara el poder calorífico de las maderas encontradas en zonas áridas de México con el de otras especies madereras utilizadas para la combustión tradicional. Con base en la identificación de especies con mayor poder calorífico y de rápido crecimiento, se pretende guiar al sector consumidor, o bien a las propias autoridades, a tomar mejores decisiones en sus políticas de reforestación y de reacondicionamiento de suelos, considerando esta aplicación como fuente de energía. Hasta donde se conoce, estas plantas de zonas áridas no tienen antecedentes de la determinación del poder calorífico o del estudio de sus cenizas. En contraste, y con resultados promisorios desde el punto de vista energético, Ngangyo-Heya et al. (2016) y Martínez, Pedraza-Bucio, Orihuela, López y Rutiaga (2014) han estudiado maderas en México, desde aquellas provenientes de zonas semiáridas, hasta aquellas de zonas boscosas. Las maderas de zonas áridas pueden tener otras aplicaciones de gran valor agregado, mismas que deben considerarse para una mejor toma de decisiones en la búsqueda de analizar la utilidad de tales especies. Las cenizas de cada madera fueron analizadas por medio de la técnica de XPS para estimar la composición de las maderas, esto con el fin de contrastar su sustentabilidad en relación con las cenizas de carbón, que en ocasiones suelen contener concentraciones de metales pesados que explican la no sustentabilidad de tales combustibles.

\section{OBJETIVOS}

Este estudio tiene como objetivo conocer los poderes caloríficos de maderas típicas de las zonas áridas del centro del país, mismas que, en un alto porcentaje y en altos volúmenes, son utilizadas por la población de esas regiones para la combustión y cocción de alimentos. Adicionalmente, se compara el poder calorífico de las maderas encontradas en zonas áridas de México con el de otras especies de madera utilizadas para la combustión tradicional. Con base en los resultados de identificación de especies con mayor poder calorífico y rápido crecimiento se pretende guiar al sector consumidor, o bien, a las propias autoridades, para mejorar la toma de decisiones en las políticas de reforestación y de reacondicionamiento de suelos que definan.

\section{MATERIALES Y MÉTODOS}

Se analizaron muestras de maderas de maguey (Agave salmiana), huizache (Acacia farmesiana), nopal (Opuntia streptacantha), garambullo (Myrtillocactus geometrizans), y cardón (Pachycereus sp). Las muestras fueron recolectadas en el ejido de Tezontepec de Aldama, Estado de Hidalgo, 
México, en el otoño de 2017. El lugar se caracteriza por ser un sitio de tierra árida con una precipitación pluvial menor a $350 \mathrm{~mm} /$ año. En el caso de las plantas, se seleccionó el área de mayor volumen de cada especie para obtener una muestra en forma de aserrín de esta parte. Luego, el aserrín se secó en una estufa a $120^{\circ} \mathrm{C}$ durante 3 h; por último, se trituró el material en un mortero de ágata hasta lograr el tamaño más fino posible, enseguida se procedió a pasar la muestra a través de un tamiz de $600 \mu \mathrm{m}$, misma que se guardó y se conservó en un desecador a temperatura ambiente. Posteriormente, se tomó una muestra del orden de los $5 \mathrm{mg}$ de cada madera y bajo flujo de aire se metió en un equipo de análisis termogravimétrico (ATG) y de análisis térmico diferencial (ATD) de la marca (Thermo Scientific Instrument, modelo TA). Para conocer la pérdida de peso y el flujo de calor de cada proceso que implica absorción de energía hasta la combustión de las maderas, los polvos se trataron térmicamente con una velocidad de calentamiento de $10{ }^{\circ} \mathrm{C} \mathrm{min}^{-1}$ desde la temperatura ambiente hasta $950{ }^{\circ} \mathrm{C}$. Por otro lado, se llevó a cabo la evaluación del calor de reacción mediante el análisis termoquímico empleando los datos del análisis ATD tomando en cuenta la constante de calibración de crisol de referencia y las áreas bajo la curva de cada proceso exotérmico y restando, cuando fue el caso, el área del pico endotérmico de las muestras. En ese sentido, el área medida neta $\mathrm{A}$ es proporcional al cambio de entalpia $\Delta \mathrm{H}$ para el proceso térmico, según la expresión $\Delta \mathrm{H}$ $=\mathrm{A} \cdot \mathrm{K} / \mathrm{m}$, donde $\mathrm{m}$ es la masa de la muestra y $\mathrm{K}$ es un factor de calibración, tal como lo han mostrado Magdziarz y Wilk, (2013). El poder calorífico es la suma de los valores absolutos de la entalpía y se distingue entre el poder calorífico superior y poder calorífico inferior según se produzca o no la condensación de agua en la reacción. En este caso, se estima el calor de combustión que es igual al poder calorífico superior.

Para fines de comparación se estimó el poder calorífico superior en base seca empleando una bomba calorimétrica IKA C6000 a volumen constante siguiendo el protocolo de medición estándar de acuerdo con la norma alemana DIN 51900-1:2000 (din 51900-1:2000) (Soto y Núñez, 2009). En este caso, se colocaron en el crisol $100 \mathrm{mg}$ de muestra y se generó la combustión con un filamento de ignición. A partir del calor liberado (Q) por la masa del sistema (bomba, accesorios y agua utilizada) debido a la combustión de la madera a volumen constante y usando factores de corrección para condiciones de temperatura ambiente, se obtiene el poder calorífico $\mathrm{PC}=(\mathrm{Q}-\mathrm{q}) / \mathrm{m}$; q es la corrección por el calor generado por el filamento de ignición y formación de compuestos de nitrógeno y azufre (que en este caso son despreciables).

Por otra parte, las cenizas de cada madera fueron analizadas por medio de la técnica de espectroscopía fotoelectrónica de rayos X (XPS). El análisis se realizó en las muestras seleccionadas utilizando un sistema de barrido de ultra alto vacío (UHV) PHI 5000 VersaProbe II con una fuente de rayos X monocromática $\mathrm{Al} \mathrm{K} \alpha(\mathrm{h} \nu=1486.6 \mathrm{eV}$ ) de un diámetro de haz de $100 \mu \mathrm{m}$ y un analizador MCD. Los espectros XPS se obtuvieron a $45^{\circ}$ de la superficie normal en la energía de paso constante (CAE) $\mathrm{E}_{0}=117.40$ y $11.75 \mathrm{eV}$ para la superficie de levantamiento y la exploración estrecha de alta resolución. Las muestras de superficie se grabaron durante 8 minutos con $1 \mathrm{kV} \mathrm{Ar}^{+}$a $0.25 \mu \mathrm{A} / \mathrm{mm}^{2}$. Las energías de unión se corrigieron para el cambio de carga utilizando el photopeak $3 \mathrm{~d} 5 / 2$ de plata a $368.20 \mathrm{eV}$, con un FWHM de $0.56 \mathrm{eV}$, y grupos de hidrocarburos C 1s a $285.00 \mathrm{eV}$, Au 4f 7/2 en $84.00 \mathrm{eV}$ en la posición del nivel central del núcleo central. El análisis de deconvolución XPS de los espectros se ajustó a los programas PHI Multipak@ versión 9.6.0.15 y SDPC v 4.1. La incertidumbre asociada de la energía de unión de la deconvolución del pico central fue de alrededor de 5\% ( \pm $0.05 \mathrm{eV}$ ) y un valor de $\chi^{2}$ menor a 1.5 .

\section{RESULTADOS Y DISCUSIÓN}

En la figura 1 se registran los análisis térmicos de especies representativas de maguey, huizache, nopal y garambullo en los que se puede apreciar degradación térmica multietapa. $\mathrm{El}$ análisis ATG-ATD en el intervalo de $50{ }^{\circ} \mathrm{C}$ a $100{ }^{\circ} \mathrm{C}$ se asocia a pérdida de peso debido a humedad que para las maderas de huizache, nopal y garambullo fue de aproximadamente $5 \%$ y para maguey $8 \%$. En el intervalo de $100{ }^{\circ} \mathrm{C}$ a $200{ }^{\circ} \mathrm{C}$ hay una relativa estabilidad térmica con 
pérdida de masa de apenas $1 \%$ adicional en las maderas, excepto para el maguey (Fig. 1a) que ha perdido aproximadamente $6 \%$ de masa adicional en este intervalo de temperatura, probablemente debido a deshidratación de hemicelulosa (Jiménez-Muñoz, Prieto-García, PrietoMéndez, Acevedo y Rodríguez, 2016). El principal y más importante cambio asociado a pérdida de peso ocurre en el intervalo entre $250{ }^{\circ} \mathrm{C}$ y $350^{\circ} \mathrm{C}$ para todas las maderas. Para nopal, huizache y garambullo la pérdida es de alrededor de $54 \%$ (Fig. 1b-d) y es menor para el maguey con 37\% de pérdida en este intervalo de temperatura (Fig. 1a). Esta disminución de masa se ha asociado principalmente a degradación térmica de la celulosa, que en el bagazo de caña o para la cascarilla de café es de $44 \%$ entre $200{ }^{\circ} \mathrm{C}-400{ }^{\circ} \mathrm{C}$ (Jiménez-Muñoz, et al., 2016) y para el bagazo de $A$. tequilana es de $63.8 \%$ a $360{ }^{\circ} \mathrm{C}$ (Naranjo, et al., 2016). Los cambios termogravimétricos observados entre $350^{\circ} \mathrm{C}$ y 450 ${ }^{\circ} \mathrm{C}$ se han atribuido a la deshidratación de lignina. Los porcentajes en masa descendieron desde 10\% para maguey (Fig. 1a) y hasta $26 \%$ para huizache (Fig. 1b). Finalmente, para el intervalo de temperatura entre $450{ }^{\circ} \mathrm{C}$ y $650{ }^{\circ} \mathrm{C}$, los cambios térmicos son atribuidos a la degradación de lignina y fragmentación de celulosa.
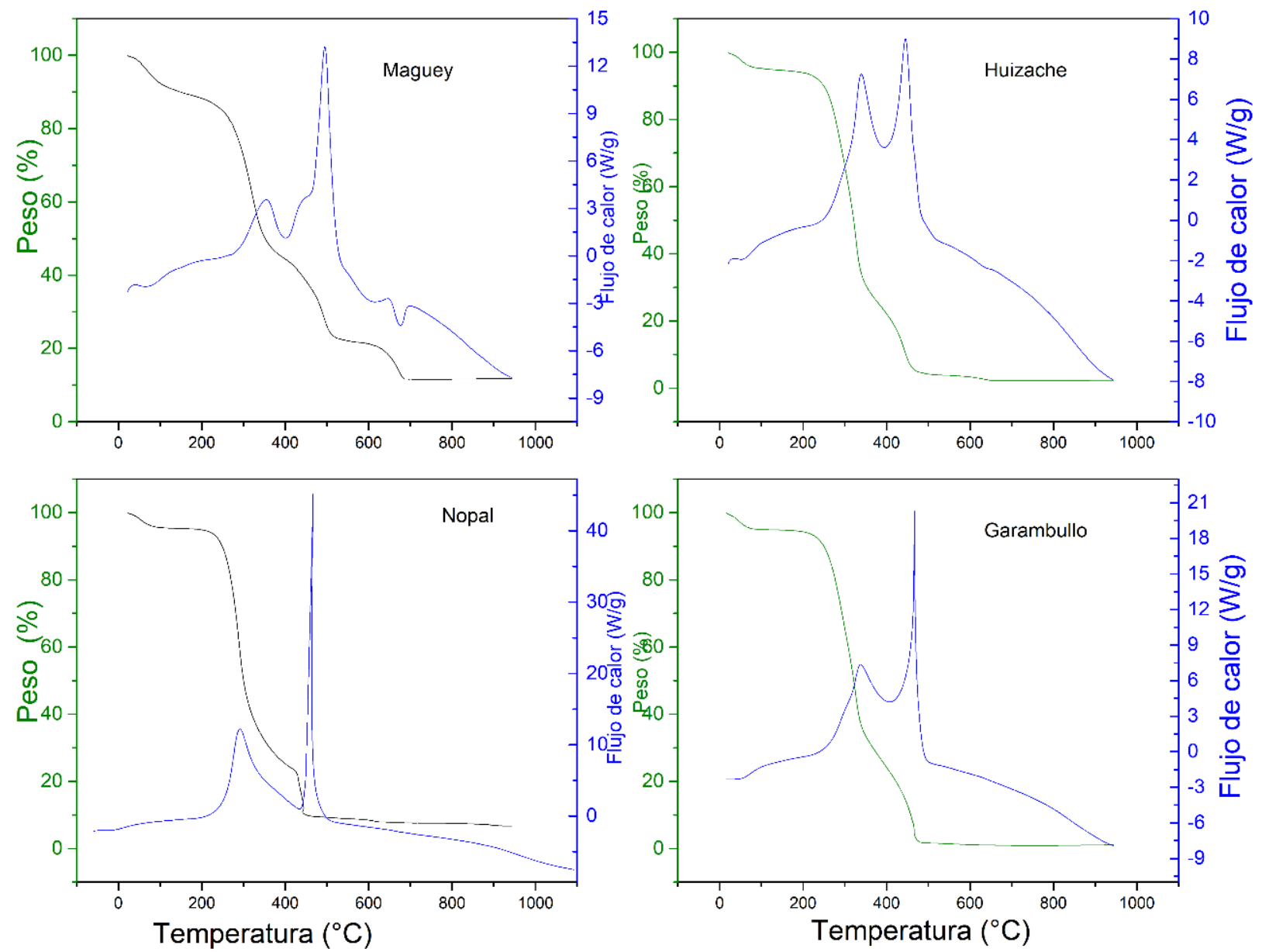

FIgURA 1. Termogramas y curvas ATD de las maderas de a) maguey, b) huizache, c) nopal y d) garambullo como muestras representativas del estudio.

La curva que inicia con altos valores corresponde a la de pérdida de peso y la menor a la de ATD. 
Las maderas que presentaron los valores menores y mayores de pérdida de peso fueron nopal $(2 \%)$ y maguey (19\%), mientras que para huizache fue $11 \%$ y garambullo $14 \%$. Al respecto, se ha informado que en cascarilla y paja del cultivo de cebada los contenidos de lignina son de $10.6 \%$ a $19.7 \%$, que por cierto son valores mucho más altos que los informados para agaves cubanos (desde 3.1\% a 9.9\%). Es conocido que el contenido de lignina y el poder calorífico están fuertemente correlacionados (Acar y Ayanoglu, 2016). Por esta razón, los materiales lignocelulósicos son ampliamente utilizados por sus propiedades energéticas (Musule, et al., 2016). Para temperaturas mayores a $650{ }^{\circ} \mathrm{C}$ la disminución de peso se atribuye a la degradación de compuestos inorgánicos, el cual es mayor en el garambullo, ya que a esta temperatura se ha perdido prácticamente todo el peso de la muestra. En general, los comportamientos de pérdida de peso en función de la temperatura para todas las maderas fueron muy similares, excepto para el maguey, donde se observó mayor pérdida de peso en el intervalo de $50{ }^{\circ} \mathrm{C}$ a $250{ }^{\circ} \mathrm{C}$; después, de $250^{\circ} \mathrm{C} \mathrm{a} 650^{\circ} \mathrm{C}$ la pérdida de peso fue menor en comparación con las otras maderas.

En general, en el ATD de las maderas se observaron múltiples curvas exotérmicas (picos crecientes) y una o dos endotérmicas (decrecientes), para el caso del maguey. Por ejemplo, en la figura 2 se muestran las principales entalpías de combustión por unidad de masa para maguey. Estas corresponden a las transformaciones termoquímicas de los componentes de la madera llevadas a cabo a diferentes temperaturas que implican procesos de absorción de energía. La primera transición endotérmica es muy débil y se atribuye principalmente a la evaporación de agua en las muestras $\left(25^{\circ} \mathrm{C}-120^{\circ} \mathrm{C}\right)$. A temperaturas $>200{ }^{\circ} \mathrm{C}$ se inicia la deshidratación y posterior descomposición de los componentes de la madera, tales como celulosa y lignina, en la que se observan varias reacciones exotérmicas, estas últimas relacionadas con la formación de productos gaseosos liberados durante pirolisis. En particular, se identificaron dos principales picos exotérmicos para maguey localizados en $349.9^{\circ} \mathrm{C}$ y $495.9^{\circ} \mathrm{C}$, así como uno endotérmico en aproximadamente $700{ }^{\circ} \mathrm{C}$, que, mediante integración del área bajo la curva, permite estimar la entalpía másica de esta madera que alcanza los $10050 \mathrm{~J} / \mathrm{g}$ (Fig. 2). A partir de cada curva de ATD correspondiente a cada material estudiado, y realizando la integración de las áreas bajo las curvas de los picos exo como endotérmicos detectados, se obtiene el poder calorífico de las maderas que se resumen en la tabla 1 y, para fines de comparación, también se informan los valores del poder calorífico estimados con la bomba calorimétrica en este trabajo y, cuando fue posible, los estimados en la literatura (Chávez e Hinojosa, 2010; Mejía, 2016).

Los valores de los poderes caloríficos estimados por análisis ATD en alguno de los casos, como el nopal, caen dentro de orden de magnitud de los valores del PC medido con bomba calorimétrica, pero en general, los PC de las muestras son menores que los calculados con la bomba IKA debido a la incertidumbre experimental ( $\pm 5 \%$ ) asociada a las medidas. Los valores de los poderes caloríficos estimados en este trabajo están por debajo de los informados en la literatura. Esta corresponde a estudios realizados fuera de México (Mejía, 2016), o que podrían corresponder a muestras de otras variedades de cactus de zonas áridas o sin identificación de la especie (Chávez e Hinojosa, 2010); muestras que se cortaron en diferentes partes de la planta como el fuste o las ramas, (Mejía, 2016) o muestras que fueron previamente pirolizadas (Chávez e Hinojosa, 2010) o comprimidas. Como puede apreciarse de la tabla 1, los valores estimados en el presente estudio para el poder calorífico de Agave salmiana solo son compatibles con los valores informados para bagazo de la misma especie (Chávez e Hinojosa, 2010). En ambos casos se utilizó el método DTA y las condiciones de preparación para el análisis de las muestras fueron similares. 


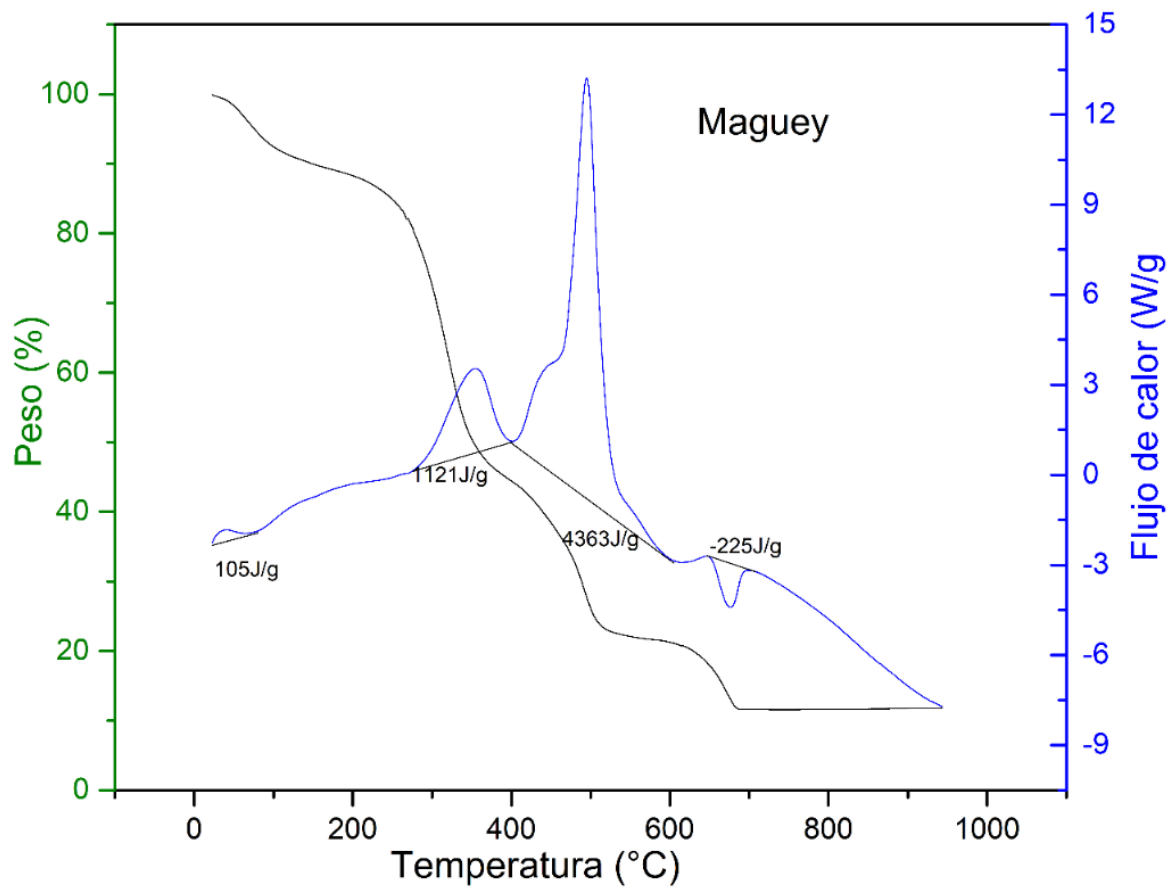

Figura 2. Análisis térmico de la curva ATG-ATD de maguey, como material representativo de este estudio.

Este análisis permite la determinación del área bajo la curva la cual es proporcional al área de los picos exotérmicos y endotérmicos para estimar el poder calorífico. La suma total por la constante del equipo da los valores finales que se resumen en la tabla 1.

TABLA 1. Poder calorífico para diferentes maderas estimado en este trabajo por análisis ATD y con bomba calorimétrica, así como los informados en la literatura.

\begin{tabular}{|c|c|c|c|c|}
\hline Madera & $\begin{array}{c}\text { PC (J/g) Análisis } \\
\text { ATD }\end{array}$ & $\begin{array}{c}P C(J / g) \\
\text { Bomba } \\
\text { calorimétrica }\end{array}$ & $\begin{array}{c}P C(J / g) \\
\text { Literatura }\end{array}$ & Referencia \\
\hline \multirow{2}{*}{ Nopal } & \multirow{2}{*}{11275} & \multirow{2}{*}{11300} & $16093-17556^{a}$ & Acar y Ayanoglu (2016) \\
\hline & & & $14379^{b}$ & Mejía (2016) \\
\hline \multirow[t]{2}{*}{ Garambullo } & 10578 & 11405 & ---- & --- \\
\hline & & & $8400^{c}$ & Mejía (2016) \\
\hline \multirow[t]{3}{*}{ Maguey } & 10050 & 10500 & $9550^{d}$ & Mejía (2016) \\
\hline & & & $19360^{e}$ & Mejía (2016) \\
\hline & & & $18540^{f}$ & Mejía (2016) \\
\hline \multirow[t]{2}{*}{ Huizache } & 11420 & 11605 & $19269^{9}$ & Mejía (2016) \\
\hline & & & $18898^{h}$ & Ngangyo-Heya et al. (2016) \\
\hline Cardón & 9275 & 9926 & - & - \\
\hline
\end{tabular}

a, muestra en base seca, no se identificó la especie; b, muestra en base seca de Opuntia ficus; c, fibra de Agave salmiana; d, bagazo de Agave salmiana; e, muestra pirolizada de Agave salmiana; f, pastillas comprimidas de Acacia pennatula; g, biomasa en base seca de Acacia famesiana recolectada en Honduras, seleccionada del fuste; h, biomasa en base seca de Acacia famesiana recolectada en Honduras, seleccionada de las ramas. 
En general, el valor del poder calorífico registrado en la literatura para diferentes especies analizadas en condiciones similares varía en aproximadamente $8 \%$. Por ejemplo, el poder calorífico medio citado para diferentes Acacia es de $19410 \mathrm{~J} / \mathrm{g} \pm 1530 \mathrm{~J} / \mathrm{g}$. Estos valores dependen de la especie, las condiciones de crecimiento, parte y posición del árbol donde las muestras fueron seleccionadas, edad, humedad y otros muchos factores. Se puede destacar que los valores del PC de nopal (11 $300 \mathrm{~J} / \mathrm{g})$; garambullo (11 405 J/g); huizache (11 605 J/g); maguey $(10500 \mathrm{~J} / \mathrm{g})$ y cardón (9926 J g) crecidas en la zona centro de Hidalgo, México y bajo las condiciones específicas de preparación mencionadas, no habían sido informados previamente en la literatura. Hay casos en donde, a pesar de que los valores del PC de las maderas son menores que los registrados en la literatura, se sugiere su aplicación como combustible en zonas áridas (Tabla 2). Esta aseveración se basa en que los poderes caloríficos obtenidos en este trabajo son mayores a los valores comparados con otros materiales combustibles como el bagazo húmedo, la corteza escurrida, coseta de caña, serrín húmedo, briquetas y desechos sólidos (Tabla 2) (Mejía, 2016 y Ngangyo et al., 2016).

Como se mencionó anteriormente, se ha informado de una fuerte correlación entre el poder calorífico y la biomasa de lignocelulosa. Por tal razón, en la figura $3 \mathrm{a}$ se ha registrado el poder calorífico vs el porcentaje de lignocelulosa (asociada principalmente a lignina y celulosa) estimada por análisis ATG-ATD para nopal, huizache, garambullo y maguey. Como puede observarse, en la figura 3a hay un incremento proporcional entre el PC vs \% lignocelulosa para nopal y huizache y después decae para garambullo y maguey. Para explicar este decaimiento del poder calorífico con el aumento de lignocelulosa, se considera el porciento de carbohidratos en la muestra de biomasa. $\mathrm{Al}$ respecto, se ha informado que una especie rica en carbohidratos tendrá un PC más bajo que el promedio de otras maderas (Castilho, 1984; Ibarra y Rueda, 2018). En consecuencia, la proporción entre lignocelulosa y carbohidratos tiene una fuerte relación con el PC. Si definimos el cociente $r=\%$ lignocelulosa/\% carbohidratos y graficamos el PC $v$ s este cociente, se puede explicar el comportamiento del PC para las diferentes maderas (Fig. 3b). De acuerdo con esta figura, el mayor poder calorífico de la madera de huizache se debe a que el cociente $r$ fue el más bajo de todos $(r=0.20)$ y para la madera de maguey, con el más bajo poder calorífico, se debe a que el cociente $r$ fue el más alto de todos $(r=0.51)$, mientras que para el nopal el cociente $r$ fue de 0.36 . Entonces, se puede decir que para $r$ pequeña se tendría alto poder calorífico y para $r$ grande, bajo poder calorífico.

TABLA 2. Poderes caloríficos medios de biomasa

\begin{tabular}{|c|c|}
\hline Combustible & $\begin{array}{l}\text { Poder calorífico medio } \\
\mathrm{kJ} / \mathrm{kg}\end{array}$ \\
\hline Bagazo húmedo & 10500 \\
\hline Bagazo seco & 19200 \\
\hline Cáscara de cacahuate & 17800 \\
\hline Cascarilla de arroz & 13800 \\
\hline Celulosa & 16500 \\
\hline Corteza escurrida & 5900 \\
\hline Cosetas de caña & 4600 \\
\hline Madera seca & 19000 \\
\hline Madera verde $\left({ }^{*}\right)$ & 14400 \\
\hline Paja seca de trigo & 12500 \\
\hline Paja seca de cebada & 13400 \\
\hline Serrín húmedo & 8400 \\
\hline Viruta seca & 13400 \\
\hline Desechos sólidos & 5630 \\
\hline Briquetas & 7690 \\
\hline
\end{tabular}



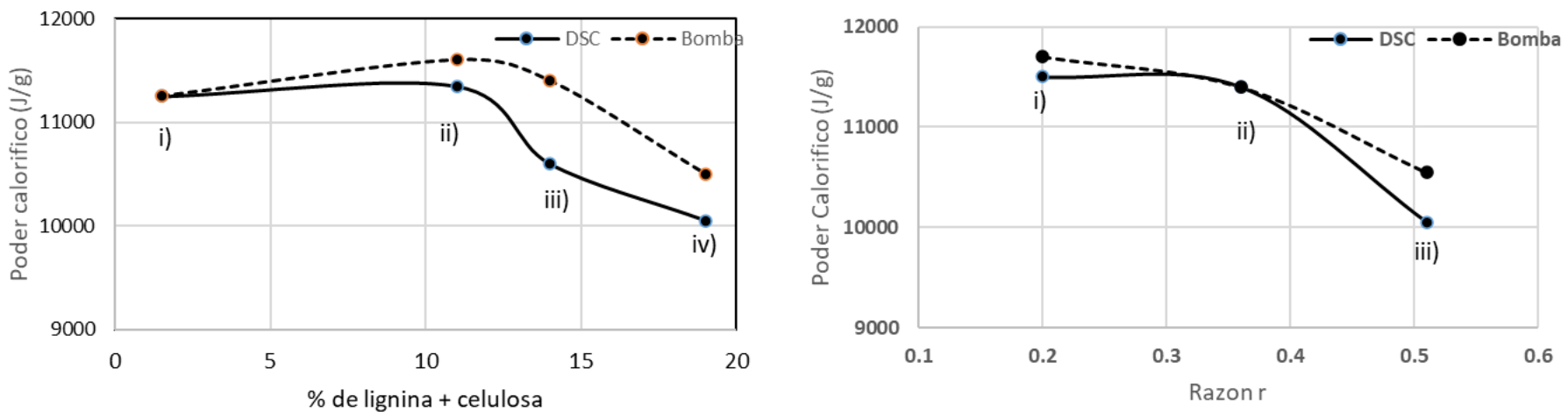

FIgURA 3. Poder calorífico vs $\%$ Lignina $+\%$ celulosa para i) nopal, ii) huizache, iii) garambullo y iv) maguey, (a). Poder calorífico vs Cociente r para i) huizache, ii) maguey y iii) nopal, respectivamente (b).

Por otra parte, se utilizó la técnica de espectroscopía XPS con la finalidad de observar posibles trazas de metales pesados en las maderas y en cenizas de nopal, maguey y garambullo. En la figura 4 se ha registrado un espectro XPS representativo de este análisis para nopal. El análisis XPS de esta madera sin quemar (Fig. 4a) muestra un diagrama de dos señales intensas a $284.0 \mathrm{eV}$ y $530.2 \mathrm{eV}$, en proporciones de $63.3 \%$ y $31.2 \%$, consistentes con energía de enlace de $\mathrm{C}$ 1s y O 1s, así como otras señales más débiles asociadas a estos mismos compuestos localizadas alrededor de esta energía de enlace principal. Otras asignaciones identificadas por las energías de enlace corresponden a $\mathrm{N}$ 1s (2.3\%), Ca 2p (1.1\%), K 2s (1\%), Si 2p (0.5\%) y Mg 1s $(0.4 \%)$. En la figura $4 \mathrm{~b}$ se muestra el XPS para las cenizas de nopal en donde las asignaciones correspondientes a las energías de enlace y los porcentajes de composición elemental fueron $\mathrm{Mg} 1 \mathrm{~s}$ (6.4\%), O 1s (47.1\%), Ca 2s (6.7\%), C 1s (37.3\%), P 2p (1.1\%), Si 2p (0.9\%), Na 1s (0.4 $\%)$. En este caso, así como en el resto de las mediciones, no se encontró evidencia de metales pesados. En la tabla 3 se registra un resumen de los elementos encontrados y su porcentaje de composición elemental relativa en cada muestra. Se puede apreciar que el contenido de carbón en la madera sin quemar es mayor en el maguey $(73.7 \%)$ y menor en el nopal (63.6\%), mientras que el contenido de carbón en ceniza fue mayor en garambullo (55.8\%) y menor porcentaje en nopal y maguey (37\%). En relación con el contenido de oxígeno, el porcentaje fue mayor en la madera de nopal (31\%) y menor en el maguey (23\%). Para las cenizas, el contenido de oxígeno fue mayor para maguey $(52 \%)$ y menor para garambullo $(36 \%)$. En principio, los sólidos provenientes de biomasa que tienen menor contenido de humedad y oxígeno aumentan su poder calorífico y producen poco humo, (García, Carrillo, Prieto, Corral y Hernández, 2007). Considerando el caso de maderas de nopal y garambullo, donde el contenido de humedad fue muy parecido (5\%), pero con menor contenido de oxígeno para garambullo (26\%), se predice mayor poder calorífico para esta madera comparado con la del nopal, tal y como se muestra en la tabla 1 para estimaciones efectuadas con la bomba IKA que el análisis ATD. En cuanto a la presencia de oxígeno, la madera de maguey fue la que tuvo la menor cantidad $(23 \%)$ en comparación con las otras maderas (entre $26 \%$ y $31 \%$ ), por lo que es de esperarse mayor poder calorífico en ella. No obstante, de acuerdo con la tabla 1, también fue la que tuvo el menor poder calorífico, aunque mayor porcentaje de humedad $(8 \%)$. Este resultado sugiere mayor importancia del porcentaje de humedad que el oxígeno contenido en las muestras para lograr altos valores de PC, por lo que es necesario mantener los valores de humedad al mínimo posible en las maderas. 


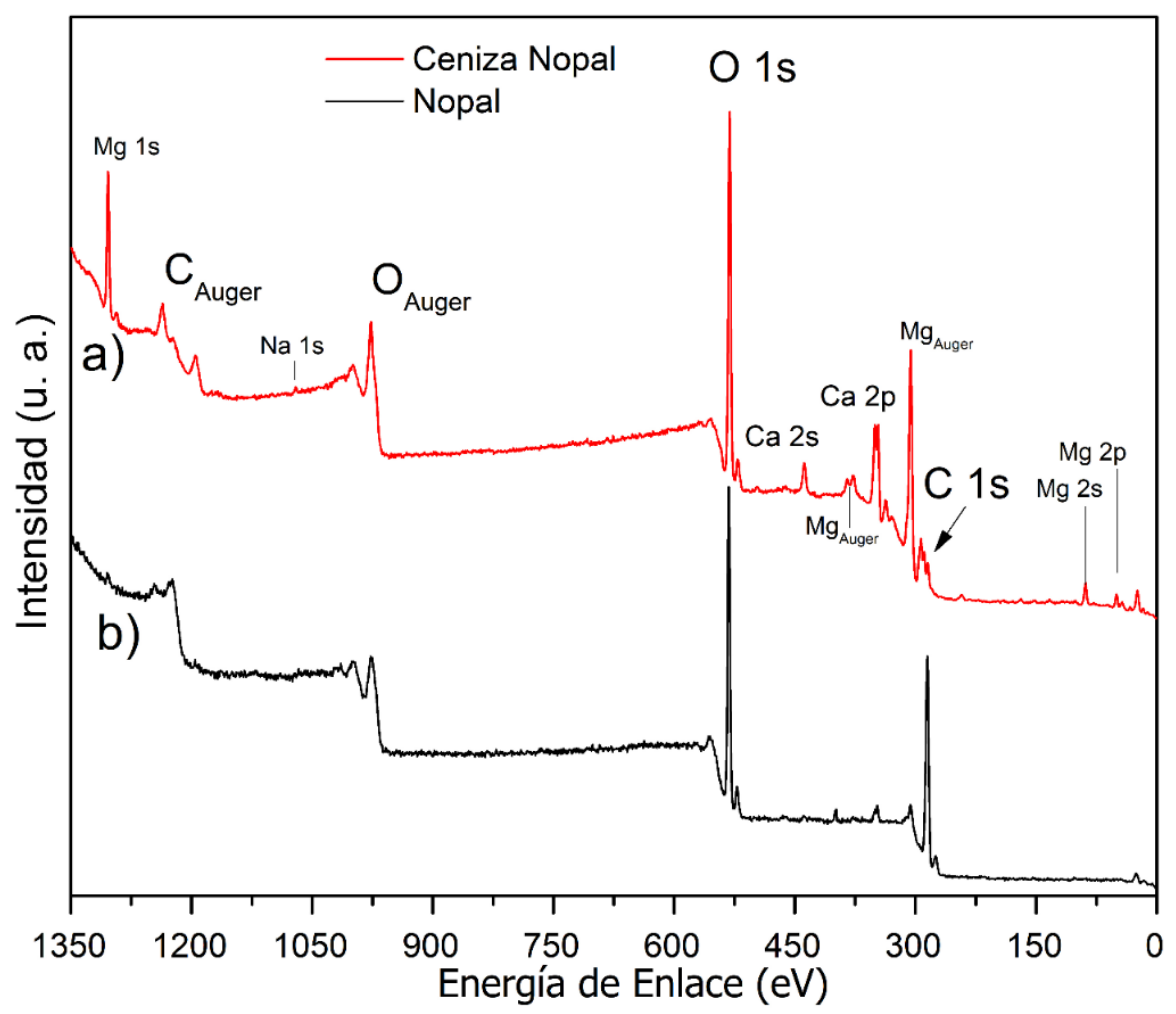

FIGURA 4. Espectro XPS de a) madera de nopal sin quemar y b) cenizas de nopal.

TABLA 3. Análisis elemental de maderas representativas y sus cenizas de nopal, maguey y garambullo medidos por la técnica de XPS.

\begin{tabular}{|c|c|c|c|c|c|c|}
\hline & $\begin{array}{c}\text { Madera de } \\
\text { nopal }\end{array}$ & $\begin{array}{c}\text { Ceniza de } \\
\text { nopal }\end{array}$ & $\begin{array}{c}\text { Madera de } \\
\text { maguey }\end{array}$ & $\begin{array}{c}\text { Ceniza de } \\
\text { maguey }\end{array}$ & $\begin{array}{l}\text { Madera de } \\
\text { garambullo }\end{array}$ & $\begin{array}{l}\text { Ceniza de } \\
\text { garambullo }\end{array}$ \\
\hline Elemento & \multicolumn{6}{|c|}{ Composición relativa (\%) } \\
\hline $\mathrm{Mg}$ & 0.4 & 6.4 & - & 5.2 & - & \\
\hline $\mathrm{O}$ & 31 & 47.1 & 23 & 52.1 & 26.2 & 36.2 \\
\hline $\mathrm{N}$ & 2.3 & - & 1.3 & - & 1.4 & - \\
\hline $\mathrm{Ca}$ & 1.1 & 6.7 & 1.7 & 3 & 0.5 & 5.2 \\
\hline$C$ & 63.6 & 37.3 & 73.7 & 37.8 & 71.4 & 55.8 \\
\hline $\mathrm{K}$ & 1 & - & 0.3 & - & - & - \\
\hline $\mathrm{Si}$ & 0.5 & 0.9 & - & 1.4 & 0.4 & - \\
\hline$P$ & - & 1.1 & - & 0.5 & - & - \\
\hline $\mathrm{Na}$ & - & 0.4 & - & - & - & - \\
\hline
\end{tabular}

Por otra parte, un indicador ambiental importante hoy en día es la intensidad de carbono, IC, (International Energy Agency [IEA] 2013), que se define como el cociente de la cantidad de carbono quemado (determinado por análisis
XPS), a la energía primaria del combustible, poder calorífico determinado con la bomba calorimétrica. Los cálculos de este parámetro, usando información de las tablas 1 y 3, permiten la estimación de este parámetro 
ambiental, que resultan ser: para la madera de nopal IC = $23 \mathrm{~g} \mathrm{C} / \mathrm{MJ}$; para la de maguey IC $=33 \mathrm{~g} \mathrm{C} / \mathrm{MJ}$ y para la madera de garambullo IC $=14 \mathrm{~g} / \mathrm{MJ}$. Este parámetro de sustentabilidad ambiental de maderas de áreas rurales y zonas áridas se contrasta con la IC propiamente del carbón comercial, que equivale a una IC clásica de $13 \mathrm{~g}$ de C/MJ. La madera de garambullo compite en este parámetro energético ambiental con un material comercial, no así la madera de maguey que se aleja mucho de este estándar para el carbón como material energético ampliamente utilizado. No obstante, debe recordarse que esta intensidad de carbono proveniente de la combustión de maderas de zonas áridas es sobre una base de sustentabilidad, propia del uso de maderas que están dentro de los ciclos naturales de la biosfera.

En resumen, se infiere, por las medidas térmicas y por el análisis XPS, que las maderas de nopal, huizache, cardón, maguey y garambullo tienen poder calorífico relativamente bajo, pero sus emisiones no alteran el medio ambiente por presencia de metales pesados durante su combustión. Además, el parámetro de la intensidad de carbono encontrado para estas maderas es comparable con otro tipo de combustibles comerciales o maderas convencionales, como lo es el carbón utilizado en la industria energética. El bajo poder calorífico, similar en algunos casos a otras maderas estudiadas en México por Ngangyo et al. (2016), Martínez et al. (2014) y A polinar, Honorato y Colotl (2017)., puede compensarse incrementando la masa de la madera en el reservorio y eliminando la mayor cantidad de humedad posible.

\section{CONCLUSIONES}

De acuerdo con los resultados obtenidos en este trabajo, se infiere que el poder calorífico de maderas que crecen en zonas áridas depende del contenido de humedad y la cantidad de oxígeno presente en su composición química y en particular de la presencia de lignina. Para el caso del nopal, maguey, garambullo y huizache, presentaron alto contenido de humedad $(5 \%-8 \%)$ y relativamente bajo poder calorífico $(10000 \mathrm{~kJ} / \mathrm{kg}-11605 \mathrm{~kJ} / \mathrm{kg}$ ). Sin embargo, su aprovechamiento para obtener energía calorífica en comunidades que habitan regiones áridas es muy común, y podría ser mejorado si las maderas son secadas por periodos prolongados a la intemperie para eliminar la mayor cantidad de humedad posible. Adicionalmente, la quema de esta biomasa no libera metales pesados al medio ambiente, por el contrario, la presencia de N, K, Ca, P y Si presentes en cenizas sugiere el aprovechamiento de estos elementos para satisfacer al menos parcialmente las necesidades de algunos nutrientes en los cultivos. Sorprendentemente, en la zona rural de México, aun hoy en día, el uso de la ceniza como un medicamento popular es una práctica común para eliminar el dolor de estómago mediante el uso de cataplasmas de ceniza aplicadas directamente al estómago. Siendo más popular el uso de la ceniza proveniente de la madera de maguey o bien la de nopal para esa acción curativa popular.

\section{REFERENCIAS}

Acar, S., \& Ayanoglu, A. (2016). Determination of higher heating values (HHVs) of biomass fuels. Energy Education Science and Technology Part A: Energy Science and Research, 28(2), 749-758.

Apolinar H., F., Honorato S., F., \& Colotl H., G. (2017). Caracterización energética de la madera de Acacia pennatula Schltdl. \& Cham. y Trema micrantha. Revista Mexicana de Ciencias Forestales, 8(39), 71-82. doi: $10.29298 /$ rmcf.v8i39.44

Banco Mundial [BM] (2018). Indicadores. Promedio detallado de precipitaciones ( $\mathrm{mm}$ anuales), Washington, Recuperado de: https://datos.bancomundial.org/indicador/AG.LND.PRCP.M M.

Castilho, M. (1984). Determinación del poder calorífico de 20 especies forestales de la amazonia peruana. Revista Forestal del Perú, 12(1-2), $1-15$.

Chávez G., L., \& Hinojosa, M. (2010). Bagasse from the mezcal industry as an alternative renewable energy produced in arid lands. Fuel, 89(12), 4049-4052. doi: 10.1016/j.fuel.2010.07.026

García B., V., Carrillo P., A., Prieto R., J. A., Corral R., J., \& Hernández D., J. (2007). Química de la biomasa vegetal y su efecto en el rendimiento durante la torrefacción, Revista Mexicana de Ciencias Forestales, 7(38), 5-24.

Greenpeace (2020). ¿Qué es la pobreza energética y por qué la sufrimos en México?, México. Recuperado de https://www.greenpeace.org/mexico/blog/8406/que-es-lapobreza-energetica-y-por-que-la-sufrimos-en-mexico 
Ibarra B., J. C., \& Rueda O., Y. J. (2018). Biomasa para el aprovechamiento energético. Una revisión de la caracterización y los modelos por descomposición termoquímica. Research Group on Energy and Environment, GIEMA. Universidad Industrial de Santander, Bucaramanga, Colombia. Recuperado de https://www.researchgate.net/publication/324223970_Biomasa _para_el_aprovechamiento_energetico_Una_revision_de_la_car acterizacion_y_los_modelos_por_descomposicion_termoquimica

Instituto Nacional de Estadística y Geografía [Inegi] (2019). Censo Nacional del Gobierno Federal 2018. Presentación de resultados generales. México. Recuperado de http://www.inegi.gob.mx

International Energy Agency [IEA] (2013). $\mathrm{CO}_{2}$ Emissions from fuel combustion. highlights. 2013 Edition, France. doi: $10.1787 / 22199446$

Jiménez-Muñoz, E., Prieto-García, F., Prieto-Méndez, J., Acevedo S., O. A., \& Rodríguez, L. R. (2016). Caracterización fisicoquímica de cuatro especies de agaves con potencialidad en la obtención de pulpa de celulosa para elaboración de papel. Dyna, 83(197), 232242. doi: $10.15446 /$ dyna.v83n197.52243

Kamble, S. M., Prashant D., R.C. Ranveer, R. C., \& Sahoo, A. K. (2017). Nutritional Importance of Cactus: A review. Trends in Biosciences, 10(37), 7668-7677

Magdziarz, A., \& Wilk, M. (2013). Thermal characteristics of the combustion process of biomass and sewage sludge. Journal of Thermal Analysis and Calorimetry, 114, 519-529. doi: 10.1007/s10973-012-2933-y

Martínez P., R., Pedraza-Bucio, F., Orihuela, R., López, P., \& Rutiaga Q., J. (2014). Calorific value and inorganic material for ten mexican wood species. Wood Research, 60(2), 281-292.

Mejía, P. N. (2016). Potencial biomásico y propiedades energéticas de cuatro especies del bosque seco de Honduras. TATASCAN Revista Técnica Cientifica, 26(1), 17-25.

Musule, R., Alarcón-Gutiérrez, E., Houbron E., P., Bárcenas-Pazos, G. M., Pineda-López, M. R., Domínguez, Z., \& Sánchez-Velásquez, L. R. (2016). Chemical composition of lignocellulosic biomass in the wood of Abies religiosa across an altitudinal gradient. Journal of Wood Science, 62(6), 537-547. doi: 10.1007/s10086-016-1585-0

Musule, R., Acuña, E., Romero-Hermoso Osorio, L. R., Domínguez, Z., Bárcenas-Pazos, G. M., Pineda-López, M. R., Mendonça, R. T., González, M. E., \& Sánchez-Velásquez, L. R. (2018). Growing up at different altitudes: Changes in energy content of the Abies religiosa, Bioenergy Resource, 11(1), 209-218. doi: 10.1007/s12155017-9889-5

Naranjo, C. D., Alamilla-Beltrán. L., Gutiérrez-López, G. F., TerresRojas, E., Solorza-Feria, J., Yee-Madeira, H. T., Flores-Morales, A. y Mora-\& Escobedo, R. (2016). Aislamiento y caracterización de celulosas obtenidas de fibras de Agave salmiana aplicando dos métodos de extracción ácido-alcali. Revista Mexicana de Ciencias Agrícolas, 7(1), 31-43. doi: 10.29312/remexca.v7i1.368

Ngangyo-Heya, M., Foroughbahchk-Pournavab, R., Carrillo-Parra, A., Rutiaga-Quiñones, J. G., Zelinski, V., \& Pintor-Ibarra, L. F. (2016). Calorific value and chemical composition of five semi-arid Mexican tree species. Forest, 7(3), 58. doi: 10.3390/f7030058

Secretaria de Agricultura, Ganadería, Desarrollo Rural, Pesca y Alimentación [Sagarpa] (2018). Atlas agroalimentario 2012-2018, (2018), México. Recuperado de https://nube.siap.gob.mx/gobmx_publicaciones_siap/pag/201 8/Atlas-Agroalimentario-2018

Soto, G., \& Núñez, M. (2009). Fabricación de pellets de carbonilla, usando aserrín de Pinus radiata, como material aglomerante. Maderas. Ciencia y Tecnología, 10(2), 129-137. doi: 10.4067/S0718221X2008000200005

Manuscrito recibido el 07 de febrero de 2020

Aceptado el 09 de marzo de 2021

Publicado el 23 de diciembre de 2021

Este documento se debe citar como:

Barrera-Calva., E., González, F., Hernández-Pérez, C. D., Martínez, G., Huerta-Arcos, L., Rosas-Cedillo., R., \& RenteríaTapia, V. (2021). Maderas de zonas áridas de México, poder calorífico por ATG-ATD y mediante bomba calorimétrica. Madera y Bosques, 27(3), e2732106. doi: 10.21829/myb.2021.2732106

Madera y Bosques por Instituto de Ecología, A.C. se distribuye bajo una Licencia Creative Commons Atribución-NoComercialCompartirlgual 4.0 Internacional. 